The following scientific article was officially published in the Proceedings of the International Conference on Image Analysis and Recognition in 2010, in book form in the Lecture Notes in Computer Science series published by Springer Verlag. This article's citation is as follows:

Harmouche, Rola, Farida Cheriet, Hubert Labelle, and Jean Dansereau. "Articulated Model Registration of MRI/X-Ray Spine Data." Lecture Notes in Computer Science (ICIAR 2010, Part II), Vol. 6112, pp. 20-29. Springer Berlin Heidelberg, 2010.

The final publication is available at Springer via http://dx.doi.org/10.1007/978-3-642-13775-4_3

The manuscript, as accepted by the publisher, is reproduced in the following pages.

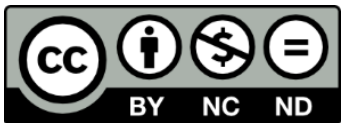

Rola Harmouche, 2010

(C) 2010 Rola Harmouche. This work is licensed under the Creative Commons AttributionNonCommercial-NoDerivatives 4.0 International License. To view a copy of this license, visit:

http://creativecommons.org/licenses/by-nc-nd/4.0/ 


\title{
Articulated model registration of MRI/X-ray spine data
}

\author{
Rola Harmouche ${ }^{1}$, Farida Cheriet $^{1}$, Hubert Labelle ${ }^{2}$, and Jean Dansereau ${ }^{1}$ \\ 1 École Polytechnique de Montréal \\ 2500, chemin de Polytechnique, Montréal, H3T 1J4 \\ 2 Hôpital Ste-Justine, \\ 3175, Chemin de la Côte-Sainte-Catherine, Montréal H3T 1C5
}

\begin{abstract}
This paper presents a method based on articulated models for the registration of spine data extracted from multimodal medical images of patients with scoliosis. With the ultimate aim being the development of a complete geometrical model of the torso of a scoliotic patient, this work presents a method for the registration of vertebral column data using 3D magnetic resonance images (MRI) acquired in prone position and X-ray data acquired in standing position for five patients with scoliosis. The $3 \mathrm{D}$ shape of the vertebrae is estimated from both image modalities for each patient, and an articulated model is used in order to calculate intervertebral transformations required in order to align the vertebrae between both postures. Euclidean distances between anatomical landmarks are calculated in order to assess multimodal registration error. Results show a decrease in the Euclidean distance using the proposed method compared to rigid registration and more physically realistic vertebrae deformations compared to thin-plate-spline (TPS) registration thus improving alignment.
\end{abstract}

\section{Introduction}

Idiopathic scoliosis is a disease of unknown cause characterized by a complex three-dimensional curvature of the spine with onset most often discovered during puberty [1]. $5.1 \%$ of adolescent girls and $3.5 \%$ of adolescent boys are affected by scoliosis, with the more severe cases requiring treatment being girls [2]. Curvature measures are usually obtained from standing X-rays on which the skeletal structures are visible. This spinal deviation in turn affects the external appearance of a scoliotic patient, which is usually characterized by a lateral trunk asymmetry and or a rib hump. Such external deformations are often aesthetically undesirable for patients and can cause psychological problems. In more severe cases, the spinal curvature can affect the physical functioning of the patient with symptoms such as chronic back problems or pulmonary problems [3]. When the spinal curvature is very pronounced surgery is necessary in order to correct some of the undesirable deformation. Surgery is most often undertaken in prone position, where a rod and screws are used to fuse the vertebrae causing the deformation. Surgeons rely on their experience and intuition in order to 
establish the adequate instrumentation that would lead to the desirable postoperative external trunk appearance. However, changes to the external shape of the trunk due to surgery are not directly related to the changes in the shape of the vertebral column. This might be due to several factors including the possible non-rigid deformation of muscles following changes in the structure of the vertebral column. As a result, the surgeon cannot predict the effects of the instrumentation on the shape of the trunk prior to completion of surgery. A geometric model combining information from the vertebral column and the surrounding soft tissue can aid in surgical planning. This additional information about soft tissue can be obtained using magnetic resonance images (MRI), which are most often acquired in prone position. Combining the X-ray and MRI data requires multimodal image registration where the images are acquired in different postures. The aim of the present work is to register MRI and X-ray data of the spine as a first step towards full MRI/X-ray registration.

So far, little work has been done on MRI/X-ray registration. Van de Kraats et al. [4] register MRI to X-ray data using fiducials manually placed on cadaveric data. The placement of fiducials is obviously not realistic in real patient data. Tomazevic et al. [5] use a novel similarity measure in order to rigidly register a series of 2D X-ray images to CT and MRI data. $11 \mathrm{X}$-ray images are required per patient for accurate results; which is not possible to acquire in normal clinical settings due to radiation issues. Registration of vertebral information from these two image modalities is difficult for two main reasons. First, obtaining 3D information of the spine from the 2D X-ray images, which is the case of the majority of X-ray systems, has only been feasible using manual intervention so far [6]. Thus, registration methods that rely on intensity information, such as mutual information for example, are not adequate for 2D X-ray to 3D MRI registration due to the lack of intensity and spatial correspondences between the two modalities. Second, with the knowledge that vertebrae are rigid structures, traditional non-rigid registration algorithms are not appropriate for the task at hand. Vertebral structures extracted from MRI data have been modeled as rigid bodies for registration purposes [7] but only using unimodal 2D MRI data. Our team has recently developed an articulated model representation for the spine using X-ray data but did not using for registration purposes [8]. This model was used by Kadoury et al. [9] in order to register a preoperative reconstructed X-ray personalized model to the intraoperative CT data of a scoliotic surgical patient. The work in [9] optimizes using Markov random fields which requires significant computation time. In addition, such a model, consisting exclusively of vertebral information, does not provide complementary information to the CT data. This exclusivity limits the application's clinical benefits. Preoperative MR images on the other hand contain soft tissue information which can be useful for surgical planning.

This paper proposes the use of articulated models for the 3D semi-rigid registration between X-ray and MRI image reconstructions. Taking into account the vertebrae's physical characteristics, they are modeled as rigid bodies, and inter-vertebral rigid transformations are calculated using local vertebral coor- 
dinate systems constructed using manually extracted landmarks. The overall transformation between the vertebrae extracted from the two image modalities is calculated from the composition of local and global transformations. In order to assess registration error, Euclidean distances between registered corresponding points using this method are compared to those obtained using rigid and thin-plate-spline registration.

This article is organized as follows: Section 2 describes the articulated model, the experimental setup, and the methods used to validate our work. The results of the proposed method are shown in section 3, followed by a conclusion in section 4 .

\section{Proposed Method}

In this work, we propose the use of articulated models in order to register a 3D reconstruction of the spine obtained from X-rays of patients with one obtained from MRIs. Vertebrae are considered as rigid bodies and inter-vertebral rigid transformations are calculated using correspondence points located on the vertebrae on each of the image modalities. In this section, the preprocessing work consisting of the 3D reconstruction of the vertebrae from medical images and the extraction of the correspondence points used for the registration will first be explained. This is followed by a description of the proposed articulated model used in order to align the vertebrae reconstructed from the MRI and X-ray

data. Finally the method used to validate the multimodal 3D alignment and to asses the whether the shape of the column is consistent in both postures will be explained.

\subsection{D reconstruction of vertebrae and point extraction}

Prior to the registration, we gathered MRI and X-ray data available at SteJustine hospital in Montreal from five patients with scoliosis for this study. In order to generate a 3D model of the spine from MRI data, T1-weighted MRI images are acquired using a Siemens Symphony system $(1.5$ Tesla, TR/TE $=$ $771 / 15,704 x 704,350$ FOV). Sagittal slices of $0.5 \mathrm{~mm}$ by $0.5 \mathrm{~mm}$ in-plane resolution and $3 \mathrm{~mm}$ thickness are acquired with a $3.6 \mathrm{~mm}$ separation between slices. The 3D shape of the seventeen thoracic and lumbar vertebrae is manually segmented from these images (figure 1(a)) and eight landmark points are manually labeled using TomoVision's SliceOmatic software so that they can be used to generate the articulated model. Those landmarks are placed on the left and right edges of the posterior, anterior, inferior and superior ends of the vertebral body for all thoracic and lumbar vertebrae (figure 1(a)).

In order to generate a 3D model of the spine from X-ray data, landmarks manually labeled on Postero-anterior and lateral radiographs are used to generate $3 \mathrm{D}$ landmark points representing the vertebral column. The 3D position of the points is obtained using an explicit calibration method and optimizing the calibration parameters with the Levenberg Marquardt method [6]. The obtained 
landmarks are used to map a generic vertebral dictionary onto the patient space. Of those landmarks, the same eight as in the case of the MRI data are used in order to generate the articulated model.
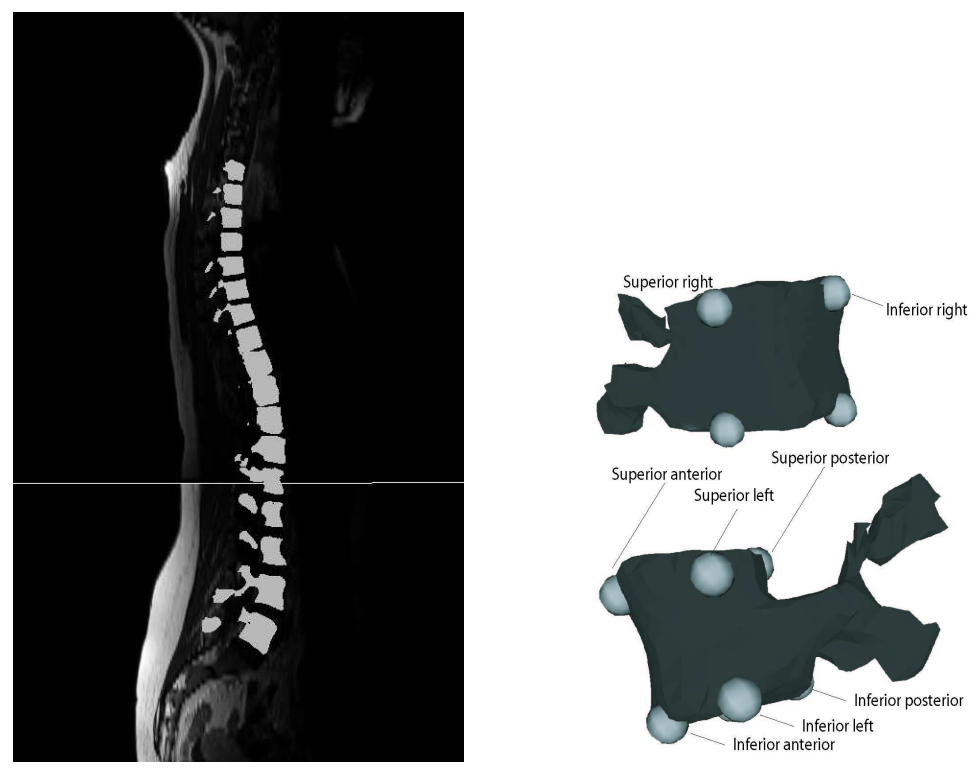

(a) MRI image and extracted vertebrae

(b) Correspondences on vertebrae

Fig. 1. 3D reconstruction of vertebrae from MRI sagittal slices along with manually labeled correspondences on each of the vertebrae.

\subsection{Articulated model deformations}

The vertebrae reconstructed from the MRI data are aligned with those of the Xray data using the articulated model proposed by Boisvert et al. [8], which models the spine as a series of local inter-vertebral rigid transformations (figure 2). Inter-vertebral transformations are first calculated on each of the image modalities separately. In order to calculate the inter-vertebral transformation $T_{i, i+1}$ from vertebra $V_{i}$ to the consecutive vertebra $V_{i+1}$, a local coordinate system is defined for each vertebra using the landmarks described above in the following manner: The 3D coordinates of landmarks on each vertebra are averaged to find the center of the coordinate system. The z-axis is defined as passing through the center from inferior to the superior end of the vertebra, the y-axis from left to right, and the $\mathrm{x}$-axis from posterior to anterior. The Gram Schmidt algorithm[10] is then used to construct an orthogonal basis from these axis forming the local coordinate systems. The position and orientation of the first vertebra 
is defined using the transformation between the absolute world coordinate system and the first vertebra's local coordinate system $\left(T_{0,1}\right)$. The intervertebral transformation matrices are then calculated as rigid transformations between the local coordinate systems of two consecutive vertebrae.

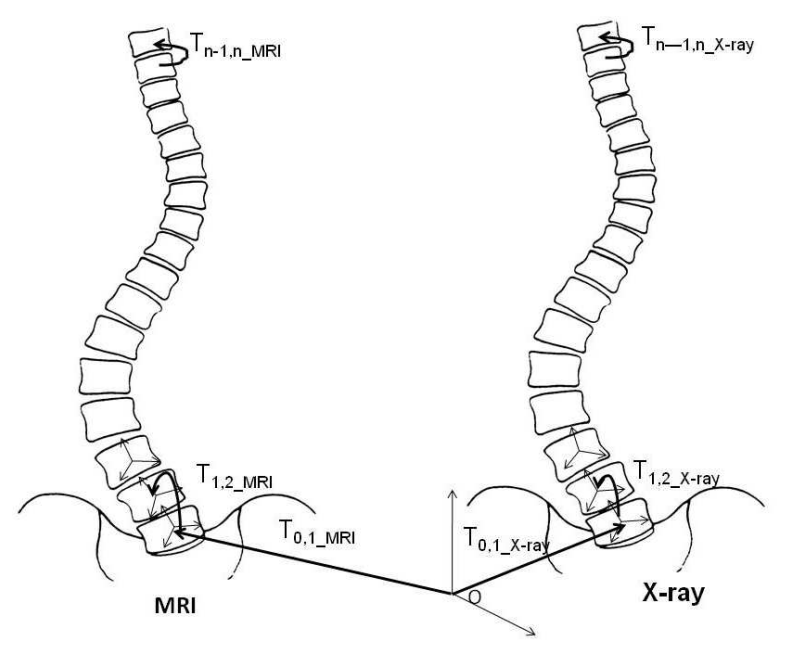

Fig. 2. Local and global transformations forming the articulated model required to align MRI onto X-ray vertebrae.

The global transformation $T_{0, i}$ between the world coordinates and the ith vertebra on each of the two image modalities is obtained by composing the local inter-vertebral transformations with the global transformation of the first vertebra in the following manner:

$$
T_{0, i}=T_{i-1, i} \circ T_{i-2, i-1} \circ T_{i-3, i-2} \circ \ldots . . \circ T_{1,2} \circ T_{0,1} .
$$

Finally, in order to register any vertebra $i$ on the MRI image $V_{i-M R I}$ to its corresponding vertebra on the X-ray data $V_{i-X-r a y}$, the inverse of the transformation from the absolute world coordinates $T_{0, i_{M} R I}$ is first applied, followed by the transformation from absolute world coordinates to $V_{i-X-r a y}$ :

$$
T_{i-M R I-X-\text { ray }}=T_{0, i_{X}-\text { ray }} \circ T_{0, i_{M} R I}^{-1} .
$$

It must be noted that the point correspondences only serve to create the articulated model and are not required during the registration process itself. The inter-vertebral transformations are obtained in the local coordinates of the vertebrae without directly relying on absolute landmark positions. The method does not require point correspondences provided that another method of obtaining the local coordinate system is used. 


\section{Results}

In order to test the validity of the articulated model registration technique, this method was compared to rigid registration and thin-plate-spline registration. Rigid registration is carried out by minimizing the least squares distance between the source and the target landmarks. In order to calculate the error for these methods, half of the landmarks were randomly selected and used for registration and the remaining ones were used for validation purposes. The registration error is then defined as the Euclidean distance between the corresponding vertebral landmarks of the registered MRI/X-ray data. This is done in order to verify whether this error is decreased using the proposed method thus signifying better alignment. Errors are reported for the thoracic and the lumbar parts of the spine separately in order to assess in which part our proposed method brings the greatest improvement.

This section will first show some qualitative results of the registered spine between MRI and X-ray using rigid, thin-plate-spline, and articulated model registration. Then, quantitative results showing registration error are shown.

\subsection{Qualitative results}

Registration results between the MRI and X-ray reconstructions are shown in figure 3(a) using rigid registration, in figure 3(b) using thin-plate-spline registration, and in figure 3(c) using our proposed articulated model registration. The landmarks used for registration are shown using spheres for the MRI data and cubes for the X-ray data. The rigid registration results show that the curvature is less pronounced at the bottom of the column in the case of the MRI (light gray) as opposed to the X-ray (dark gray) data, which can be explained by the fact that the patient is lying on a flatbed. It can be seen that our proposed method leads to more accurate alignment of the vertebrae when compared to rigid registration. The difference between the registration accuracy in the lower part of the spine can be seen in figures 3(d) and 3(f). Thin-plate-spline registration gives good alignment between the two modalities (figures 3(b) and 3(e)). However, it must be noted that non-rigid deformations such as thin-plate-splines applied to rigid structures like vertebrae misleadingly lead to smaller error values as they do not preserve their physical characteristics. In order to illustrate with an extreme case, figures $3(\mathrm{~g})$ and $(\mathrm{h})$ show an X-ray vertebra and its corresponding MRI vertebra, respectively. Even though the corresponding points are very well aligned, a misplaced landmark at the inferior end of the X-ray target vertebra caused a highly non-rigid deformation of the MRI vertebra and changed it's true physical form thus leading to erroneous results. 


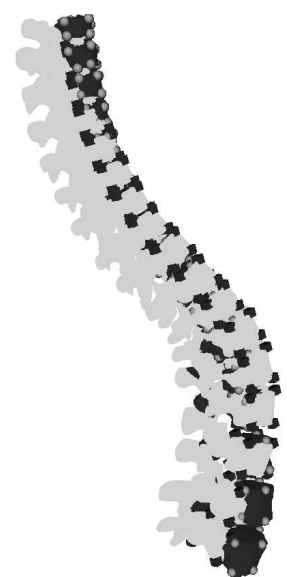

(a) Rigid registration

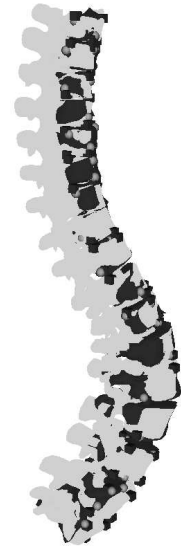

(b) TPS registration

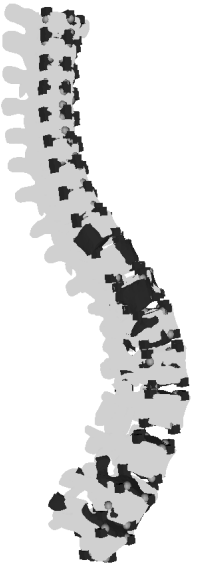

(c) Articulated model registration

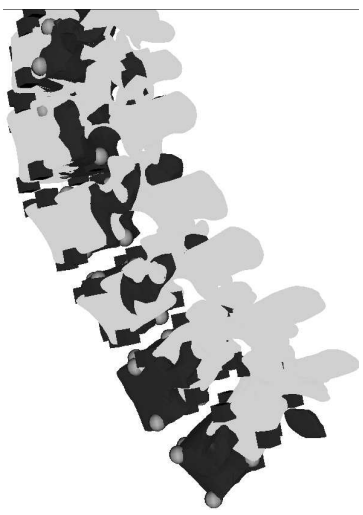

(d) Closeup of rigid registration

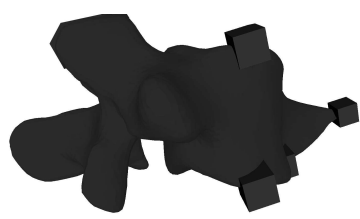

(g) an X-ray vertebra

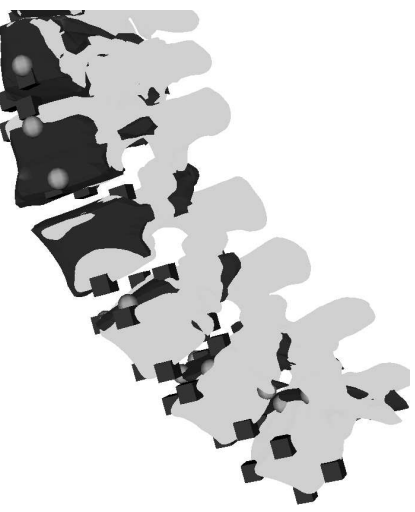

(e) Closeup of TPS registration

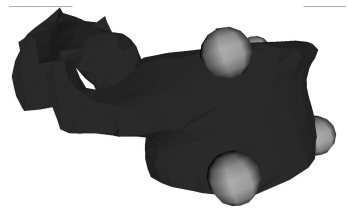

(h) corresponding MRI vertebra

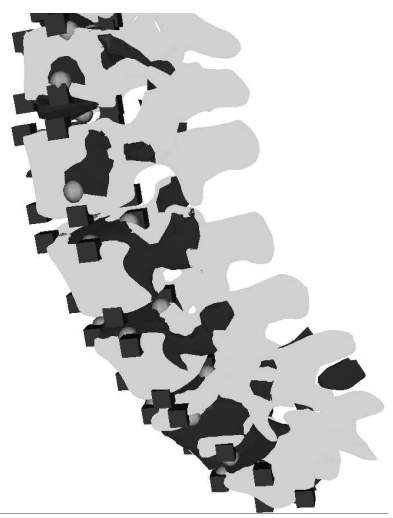

(f) Closeup of articulated model registration

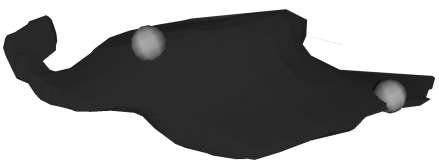

(i) TPS registered MRI vertebra

Fig. 3. Registration between vertebrae extracted from MRI (dark grey) and X-ray data (light grey). The correspondences used for the articulated model are represented by cubes (X-ray data) and by spheres (MRI data). The alignment between the vertebral column appears to be more precise using the proposed method (c) when compared to simple rigid registration (a). (d), (e) and (f) focus on the lower part of the spine where our proposed method in (f) gives particularly better results. Registration using TPS seems to yield good landmark alignment ( $b$ and e), however at the expense of non-rigidly deforming MRI vertebrae, which are rigid structures (i). 


\subsection{Quantitative results}

Table 1 shows the quantitative results for this study. The Euclidean distance between the MRI and X-ray landmarks is first calculated using our proposed articulated model method and compared to both rigid and thin-plate-spline registration for the lumbar and thoracic vertebrae together. A significant decrease in the registration error can be seen when our proposed method is used compared to rigid registration. The proposed method only provides a slight decrease in error compared to the thin-plate-spline method. Second, the results are assessed for the thoracic and lumbar areas separately. Registration errors are generally higher in the lumbar area for all methods, and, when compared with rigid registration, the improvement ratio upon use of the proposed method (rigid-proposed)/proposed is about 0.97 in both areas of the spine. When compared to thin-plate-spline registration however, the improvement ratio upon use of the proposed method drops to 0.07 overall, 0.13 in the thoracic area, and performs slightly worse in the lumbar area. However, as mentioned in the previous section, thin-plate-splines non-rigidly deform the vertebrae, which is not physically sound, as vertebrae are rigid structures. Thus, preliminary results for multimodal image registration of the spine using an articulated model to represent the deformation of vertebrae are promising when compared to simple rigid registration. This proposed method is also more physically appropriate than thin-plate-spline registration.

Table 1. Registration errors in $\mathrm{mm}$ for rigid registration ( $\mathrm{RR}$ ), thin-plate-spline registration (TPS) and for the proposed method (PM).Case five has smaller size vertebrae explaining the overall smaller error values.

\begin{tabular}{|l|l|l|l|l|l|l|l|l|l|}
\hline Case & $\begin{array}{l}\text { Overall } \\
\text { RR }\end{array}$ & $\begin{array}{l}\text { Overall } \\
\text { TPS }\end{array}$ & $\begin{array}{l}\text { Overall } \\
\text { PM }\end{array}$ & $\begin{array}{llll}\text { thoracic } \\
\text { RR }\end{array}$ & $\begin{array}{l}\text { thoracic } \\
\text { TPS }\end{array}$ & $\begin{array}{l}\text { thoracic } \\
\text { PM }\end{array}$ & $\begin{array}{l}\text { lumbar } \\
\text { RR }\end{array}$ & $\begin{array}{l}\text { lumbar } \\
\text { TPS }\end{array}$ & $\begin{array}{l}\text { lumbar } \\
\text { PM }\end{array}$ \\
\hline 01 & 12.08 & 7.18 & 9.08 & 10.79 & 6.52 & 7.21 & 15.18 & 6.52 & 13.57 \\
\hline 02 & 9.04 & 5.77 & 4.60 & 8.03 & 4.83 & 3.95 & 11.46 & 6.72 & 6.17 \\
\hline 03 & 8.12 & 3.70 & 3.17 & 6.54 & 3.22 & 3.00 & 11.91 & 4.19 & 3.58 \\
\hline 04 & 13.63 & 5.88 & 4.63 & 12.79 & 6.23 & 4.81 & 15.63 & 5.50 & 4.20 \\
\hline 05 & 4.45 & 3.35 & 2.52 & 3.78 & 3.15 & 2.30 & 6.09 & 3.56 & 3.07 \\
\hline mean & 9.46 & 5.18 & 4.80 & 8.39 & 4.79 & 4.25 & 12.05 & 5.30 & 6.12 \\
\hline
\end{tabular}




\section{Conclusion}

This paper described a method in order to register two 3D reconstructions of the spine of patients with scoliosis - one obtained from X-rays and the other one obtained from MRIs. The proposed method uses an articulated model consisting of a series of rigid transformations, taking into account inter-vertebral transformations and thus providing a more accurate representation of the movement of the vertebral column when compared to rigid registration. The method also takes into account bone rigidity providing a more realistic deformation model when compared to non-rigid registration techniques. Corresponding points on the vertebrae of each image modality are extracted and used in order to build the articulated model, and the transformation between the vertebrae of the two images are obtained using a composition of transformations. Results show a decrease in the overall registration error when using the proposed method compared to simple rigid registration, and no difference in the improvement ratio between the thoracic and the lumbar area. When compared to thin-plate-spline registration, the proposed method gives a more physically accurate registration by preserving the shape of the vertebrae, which in actuality are rigid structures.

Since the landmark extraction is done somewhat manually and the extraction process is different in the two modalities, landmark localization errors are to be expected. In the case of the X-ray reconstruction, the localization error has been previously calculated to be around $2.1 \pm 1.5 \mathrm{~mm}$ [11]. The landmark localization error in the case of the MRIs is yet to be established. The automation of landmark extraction would greatly improve the multimodal registration in addition to increasing consistency and reproducibility.

The registration of the vertebral bodies serves as a preliminary step towards the multimodal image registration of MRI and X-ray data. Little work has been done in this field so far, and no other method registers MRI to X-ray data that can be acquired in a clinical setting. This registration can be used for the purpose of evaluating the effects of posture differences on the shape of the spine. It will also allow the construction of a geometric model of the torso of patients with scoliosis combining musculo-skeletal information allowing further studies in treatment techniques which would best benefit patients and improve their quality of life.

\section{References}

1. Cottalorda, and Kohler, R: Recueil terminologique de la scoliose idiopathique. In Sauramps Medical, editor, La scoliose idiopathique (sous la direction de J. Bérard et R. Kohler), 33-40 (1997)

2. M; Asher, G; Beringer, J ; Orrick, and N. Halverhout. The current status of scoliosis screening in north america, 1986 : Results of a survey by mailed questionnaire. Spine, $14: 652-662,1989$.

3. J. Roach. Adolescent idiopathic scoliosis. Orthop Clin North Am., 30 :353-365, 1999.

4. van de Kraats EB, van Walsum T, Verlaan JJ, Oner FC, Viergever MA, and Niessen WJ. Noninvasive magnetic resonance to three-dimensional rotational x-ray registration of vertebral bodies for image-guided spine surgery. Spine, 29(3) :293-7, 2004. 
5. D. Tomazevic, B. Likar, and F. Pernus. 3-D/2-D registration by integrating 2-D information in 3-D. IEEE Transactions on Medical Imaging, 25(1) :17-27, 2006.

6. F. Cheriet, J. Dansereau, Y Petit, H. Labelle, and J.A. de Guise. Towards the selfcalibration of a multi-view radiographic imaging system for the $3 \mathrm{D}$ reconstruction of the human spine and rib cage. International Journal of Pattern Recognition and Artificial Intelligence, 13(5) :761-779, 1999.

7. J. A. Little, D. L. G. Hill, and D. J. Hawkes. Deformations incorporating rigid structures. In Proceedings of the Workshop on Mathematical Methods in Bio- medical Image Analysis, pages 104-13, San Francisco, CA, 1996. IEEE Comput. Soc. Press.

8. Boisvert, J. and Pennec, X. and Labelle, H. and Cheriet, F. and Ayache, N., Principal Spine Shape Deformation Modes Using Riemannian Geometry and Articulated Models. AMDO06, 2006, pp 346-355

9. Samuel Kadoury and Nikos Paragios,Surface/Volume-Based Articulated 3D Spine Inference through Markov Random Fields. MICCAI (1) 2009: 92-99

10. Golub, G. H. and Van Loan, C. F. Matrix Computations, 3rd ed. Baltimore, MD: Johns Hopkins, 1996.

11. Delorme, Sbastien; Labelle, Hubert; Poitras, Benot; Rivard, Charles-H.; Coillard, Christine; Dansereau, Jean. Pre-, Intra-, and Postoperative Three-Dimensional Evaluation of Adolescent Idiopathic Scoliosis. Journal of spinal disorders. Volume 13(2), April 2000, pp 93-101 